Valentino Tosatti

\title{
Limits of Calabi-Yau metrics when the Kähler class degenerates
}

Received December 6, 2007 and in revised form May 29, 2008

\begin{abstract}
We study the behavior of families of Ricci-flat Kähler metrics on a projective CalabiYau manifold when the Kähler classes degenerate to the boundary of the ample cone. We prove that if the limit class is big and nef the Ricci-flat metrics converge smoothly on compact sets outside a subvariety to a limit incomplete Ricci-flat metric. The limit can also be understood from algebraic geometry.
\end{abstract}

Keywords. Calabi-Yau manifolds, Ricci-flat metrics, degenerate complex Monge-Ampère equations

\section{Introduction}

Einstein metrics, namely metrics with constant Ricci curvature, have been an important subject of study in the field of differential geometry since the early days. The solution of the Calabi Conjecture given by Yau [Y1] in 1976 provided a very powerful existence theorem for Kähler-Einstein metrics with negative or zero Ricci curvature (the negative case was also done independently by Aubin $[\mathrm{Au}]$ ). This produced a number of nonhomogeneous examples of Ricci-flat manifolds. These spaces have been named Calabi-Yau manifolds by the physicists in the eighties, and have been thoroughly studied in several different areas of mathematics and physics. Prompted by the physical intuition of mirror symmetry, mathematicians have studied the ways in which Calabi-Yau manifolds can degenerate when they are moving in families. In general both the complex and symplectic (Kähler) structures are changing, and the behavior is not well understood. In this paper we will consider the case when the complex structure is fixed, and so we will be looking at a single compact projective Calabi-Yau manifold. The Kähler class is then allowed to vary inside the ample cone. As long as the class stays inside the cone, the corresponding Ricci-flat metrics vary smoothly, but they will degenerate when the class approaches the boundary of the cone. We will try to understand this degeneration process and see what the limiting space looks like.

To introduce our results, let us fix some notation first. Let $X$ be a compact projective Calabi-Yau manifold, of complex dimension $n$. This is by definition a projective manifold

V. Tosatti: Department of Mathematics, Harvard University, 1 Oxford Street, Cambridge, MA, 02138, USA; e-mail: tosatti@ math.harvard.edu

Mathematics Subject Classification (2000): Primary 32Q25; Secondary 14J32, 14J28, 32Q20 
such that $c_{1}(X)=0$ in $H^{2}(X, \mathbb{R})$. The real Néron-Severi space is by definition

$$
N^{1}(X)_{\mathbb{R}}=\left(H^{2}(X, \mathbb{Z})_{\text {free }} \cap H^{1,1}(X)\right) \otimes \mathbb{R}=N^{1}(X)_{\mathbb{Z}} \otimes \mathbb{R},
$$

and we assume that

$$
\operatorname{dim} N^{1}(X)_{\mathbb{R}}=\rho(X)>1 .
$$

This cohomology space contains $\mathcal{K}_{N S}$, the ample cone, which is open. Its closure $\overline{\mathcal{K}}_{N S}$ is the nef cone. Fix a nonzero class $\alpha \in \overline{\mathcal{K}}_{N S} \backslash \mathcal{K}_{N S}$, which exists precisely when $\rho(X)>1$, and a smooth path $\alpha_{t}:[0,1] \rightarrow \overline{\mathcal{K}}_{N S}$ such that $\alpha_{t} \in \mathcal{K}_{N S}$ for $t<1$ and $\alpha_{1}=\alpha$. For any $t<1$ Yau's Theorem [Y2] gives us a unique Ricci-flat Kähler metric $\omega_{t} \in \alpha_{t}$. Fixing a smooth path of reference metrics in $\alpha_{t}$, it can be verified that the Ricci-flat metrics $\omega_{t}$ vary smoothly, as long as $t<1$. We have the following very natural

Question 1. What is the behavior of the metrics $\omega_{t}$ as $t \rightarrow 1$ ?

This question has a long history: it is a special case of a problem posed by Yau [Y3], [Y4], where the complex structure is also allowed to vary; it has been stated explicitly in this form by McMullen $[\overline{\mathrm{McM}}]$ and Wilson [W2]. Physicists have also looked at this question, roughly predicting the behavior that we will describe in Theorem 1.1 (see e.g. $[\mathrm{HW}])$. One of the reasons that makes this question interesting is that the Ricci-flat metrics are not known explicitly, except in very few cases.

A nef class $\alpha \in N^{1}(X)_{\mathbb{R}}$ is called big if $\alpha^{n}>0$. Classical results of Anderson [An], Bando-Kasue-Nakajima [BKN], Tian [Ti] and more recent results of Cheeger-ColdingTian [CCT] give a partial answer to this question when $\alpha$ is big (we will explain this in Section 3. Our main theorem, which does not rely on the previous results just quoted, gives a satisfactory answer to Question 1 in this case (see Section 2 for definitions).

Theorem 1.1. Let $X$ be a compact projective Calabi-Yau manifold, and let $\alpha \in N^{1}(X)_{\mathbb{R}}$ be a big and nef class that is not ample. Then there exist a proper analytic subvariety $E \subset X$ and a smooth incomplete Ricci-flat Kähler metric $\omega_{1}$ on $X \backslash E$, both depending only on $\alpha$, such that for any smooth path $\alpha_{t} \in \mathcal{K}_{N S}$ with $\alpha_{1}=\alpha$, the Ricci-flat metrics $\omega_{t} \in \alpha_{t}$ converge to $\omega_{1}$ in the $C^{\infty}$ topology on compact sets of $X \backslash E$. Moreover, $\omega_{1}$ extends to a closed positive current with continuous potentials on the whole of $X$, which lies in $\alpha$, and which is the pullback of a singular Ricci-flat Kähler metric on a Calabi-Yau model of $X$ obtained from the contraction map of $\alpha$. If $\alpha \in N^{1}(X)_{\mathbb{Z}}$, that is, if $\alpha=c_{1}(L)$ for some line bundle $L$, then $E$ is the null locus of $L$.

There are many interesting concrete examples of our theorem, and we will examine a few of them in Section 5. Roughly speaking, the case when $\alpha$ is nef and big corresponds to a "noncollapsing" sequence of metrics, meaning that the Gromov-Hausdorff limit has the same dimension. The "collapsing" case, when $\alpha$ is nef but not big, is much harder and we will briefly discuss it at the end of the paper. We state and prove our results for a path of classes $\alpha_{t}$, but it is immediate to see that the same results hold if instead we look at a sequence of classes $\alpha_{i}$ that converge to $\alpha$. On the other hand, our result does not say anything about the case when the classes $\alpha_{t}$ approach the boundary of the ample cone without converging to a limiting class, but moving out to infinity in $N^{1}(X)_{\mathbb{R}}$. This case 
is relevant for mirror symmetry, as it should sometimes be the mirror of a large complex structure limit. Finally, let us remark that the projectivity assumptions are only technical, and that we expect that a similar result holds when $X$ is just assumed to be Kähler, and the ample cone is replaced by the Kähler cone (see Section 6 .

The paper is organized as follows. In Section 2 we recall some definitions and results from algebraic geometry. In Section 3 we prove a uniform diameter bound and we compare our results with previous literature. In Section 4 we prove our main Theorem 1.1 We use a new Moser iteration argument to get uniform bounds, using the diameter bound from Section 3. In Section 5 we give some examples where our results apply, and recover in particular a result of Kobayashi and Todorov [KT]. Finally in Section 6 we discuss some further directions for research.

\section{Some facts from algebraic geometry}

In this section we will review some definitions and results from algebraic geometry, mainly from Mori's Program, that will be used in the proof.

Let $X$ be a compact Calabi-Yau $n$-fold, that is, a compact Kähler manifold of dimension $n$ such that $c_{1}(X)=0$ in $H^{2}(X, \mathbb{R})$. We do not insist that $X$ be simply connected. Notice that it follows that $a K_{X} \cong \mathcal{O}_{X}$ for some integer $a>0$ : in fact by Theorem 1 in [Be] a finite unramified $a: 1$ cover of $X, p: \tilde{X} \rightarrow X$, has trivial canonical bundle. But $p^{*} K_{X} \cong K_{\tilde{X}} \cong \mathcal{O}_{\tilde{X}}$ and so Lemma 16.2 in [BHPV] implies that $a K_{X} \cong \mathcal{O}_{X}$. This can be rewritten as $K_{X} \sim_{\mathbb{Q}} 0$ where $\sim_{\mathbb{Q}}$ indicates $\mathbb{Q}$-linear equivalence of Cartier $\mathbb{Q}$-divisors. For the rest of this section we will assume that $X$ is projective.

Definition 2.1. A projective variety $X$ has canonical singularities if it is normal, $r K_{X}$ is Cartier for some $r \geq 1$ and there exists a resolution $f: Y \rightarrow X$ such that

$$
r K_{Y}=f^{*}\left(r K_{X}\right)+\sum_{i} a_{i} E_{i}
$$

where $E_{i}$ ranges over all exceptional prime divisors of $f$, and $a_{i} \geq 0$.

Definition 2.2 (Wilson [W1]). A Calabi-Yau model $Y$ is a normal projective variety with canonical singularities such that $K_{Y} \sim_{\mathbb{Q}} 0$.

Let $L$ be a nef line bundle on $X$, and let $\kappa(X, L)$ be its Iitaka dimension, that is,

$$
\kappa(X, L)=m \Leftrightarrow h^{0}(X, k L) \sim k^{m} \text { for all } k \text { large enough }
$$

and $\kappa(X, L)=-\infty$ if $k L$ has no sections for all $k \geq 0$. We denote by $v(X, L)$ its numerical dimension, that is, the largest nonnegative integer $m$ such that there exists an $m$-cycle $V$ such that $\left(L^{m} \cdot V\right)>0$. It is always true that

$$
\kappa(X, L) \leq v(X, L) \leq n .
$$

Definition 2.3. If $\kappa(X, L)=v(X, L)$ we say that $L$ is good (or abundant). If the complete linear system $|k L|$ is base-point-free for some $k \geq 1$ we say $L$ is semiample. 
When $|k L|$ is base-point-free, we get a morphism $\Phi_{|k L|}: X \rightarrow \mathbb{P} H^{0}(X, k L)^{*}$ that satisfies $k L=\Phi_{|k L|}^{*} \mathcal{O}(1)$. Notice that if $L$ is big, that is, $\kappa(X, L)=n$, then it is automatically good. The following is an immediate consequence of the base-point-free theorem (Theorem 6.1.11 in [KMM]).

Theorem 2.1 (Kawamata). Assume $X$ is a projective Calabi-Yau. If L is good then it is semiample.

The next theorem is classical (see Theorem 2.1.27 in [L]).

Theorem 2.2 (Iitaka). Let $L$ be semiample. Then there exists a surjective morphism $f$ : $X \rightarrow Y$ where $Y$ is a normal irreducible variety, $f_{*} \mathcal{O}_{X}=\mathcal{O}_{Y}$, and $L=f^{*} A$ for some ample line bundle A on $Y$. In fact, $f=\Phi_{|k L|}$ for all $k$ sufficiently divisible.

We will call $f$ the contraction map of $L$. There is a version of the base-point-free theorem for Cartier $\mathbb{R}$-divisors, essentially due to Shokurov [Sh]. If $D$ is a Cartier $\mathbb{R}$-divisor on $X$ we say that $D$ is semiample if there exist a normal irreducible projective variety $Y$, a surjective morphism $f: X \rightarrow Y$ with $f_{*} \mathcal{O}_{X}=\mathcal{O}_{Y}$, and an ample $\mathbb{R}$-divisor $A$ on $Y$ such that $D \sim_{\mathbb{R}} f^{*} A$. Again we will call $f$ the contraction map of $D$. Then the following holds (Theorem 7.1 in $[\mathrm{HM}]$ ):

Theorem 2.3. Assume $X$ is a projective Calabi-Yau. If $D$ is a Cartier $\mathbb{R}$-divisor which is nef and big, then it is semiample.

The contraction map of $D$ is in fact also the contraction map of a suitable nef and big line bundle $L$ (see the proof of Proposition 4.1 below). We also have the following theorem (Theorem 5.7 in [Ka1] or Theorem 1.9 in [Ka2]).

Theorem 2.4 (Kawamata). Assume $X$ is a projective Calabi-Yau. Then the subcone of $\overline{\mathcal{K}}_{N S}$ given by nef and big classes is locally rational polyhedral.

If $L$ is a line bundle, its stable base locus is the intersection of the base loci of $|m L|$ for all $m \geq 1$. It is equal to the base locus of $|m L|$ for some $m$ (see Prop. 2.1.21 in $[\mathrm{L}])$. If $L$ is now nef and big, we define the augmented base locus of $L, \mathbf{B}_{+}(L)$, to be the stable base locus of $L-\varepsilon H$ for any ample divisor $H$ and any small enough rational number $\varepsilon>0$. This definition is well-posed (see Lemma 10.3.1 in [L]) and a theorem of Nakamaye ([N], $[\mathrm{L}]$ ) says that $\mathbf{B}_{+}(L)$ is equal to the null locus of $L$, that is, the union of all positive-dimensional subvarieties $V \subset X$ such that $\left(L^{\operatorname{dim} V} \cdot V\right)=0$.

Finally, let us state a well-known conjecture (see 10.3 of Peternell's lectures in $[\mathrm{MP}]$ ).

Conjecture 2.1. Assume $X$ is a projective Calabi-Yau. If $L$ is a nef line bundle, then $L$ is semiample.

If $L$ is effective, this conjecture follows from the log abundance conjecture. Indeed, for any small rational $\varepsilon>0$, the pair $(X, \varepsilon L)$ is klt, and the log abundance conjecture would imply that $K_{X}+\varepsilon L \sim_{\mathbb{Q}} \varepsilon L$ is semiample. 
Notice that when $X$ is a surface, Conjecture 2.1 holds: in fact, if $L$ is nef and nontrivial, then $H^{2}(X, L)=H^{0}\left(X, K_{X}-L\right)=0$ and by Riemann-Roch

$$
\operatorname{dim} H^{0}(X, L) \geq 2+\frac{1}{2} L \cdot L \geq 2,
$$

thus $L$ is effective. Then we can apply the log abundance theorem for surfaces (see e.g. $[\overline{\mathrm{FM}}]$ ) to get the result.

\section{Preliminary remarks}

In this section we will prove a uniform diameter bound and use this to compare our results to the existing literature. The diameter bound is valid in general, without any projectivity or bigness assumptions.

Let the setting be as in the Introduction, namely let $X$ be a projective Calabi-Yau $n$-fold and $\alpha \in N^{1}(X)_{\mathbb{R}}$ a big and nef class that is not ample. Given a smooth path $\alpha_{t}:[0,1] \rightarrow \overline{\mathcal{K}}_{N S}$ such that $\alpha_{t} \in \mathcal{K}_{N S}$ for $t<1$ and $\alpha_{1}=\alpha$, Yau's Theorem [Y2] gives us a unique Ricci-flat Kähler metric $\omega_{t} \in \alpha_{t}$ for any $t<1$. Then

$$
\sqrt{-1} \partial \bar{\partial} \log \frac{\omega_{t}^{n}}{\omega_{0}^{n}}=\operatorname{Ric}\left(\omega_{0}\right)-\operatorname{Ric}\left(\omega_{t}\right)=0
$$

implies that $\omega_{t}^{n}=B_{t} \omega_{0}^{n}$ for some constant $B_{t}$, which is easily computed from

$$
\alpha_{t}^{n}=\int_{X} \omega_{t}^{n}=B_{t} \int_{X} \omega_{0}^{n}=B_{t} \alpha_{0}^{n} .
$$

In particular, $B_{t}>0$ and $0<\lim _{t \rightarrow 1} B_{t}<\infty$, which means that the volume form of $\omega_{t}$ is uniformly equivalent to the volume form of $\omega_{0}$. The main theorem of this section is the following

Theorem 3.1. Let $\left(X, \omega_{0}\right)$ be a compact $n$-dimensional Ricci-flat Kähler manifold and let $\omega$ be another Ricci-flat Kähler metric such that

$$
\int_{X} \omega_{0}^{n-1} \wedge \omega \leq c_{1}
$$

for some constant $c_{1}$. Then the diameter of $(X, \omega)$ is bounded above by a constant that depends only on $n, c_{1}, \omega_{0}$.

Applying this to $\omega=\omega_{t}$ we see that the diameter of $\left(X, \omega_{t}\right)$ is uniformly bounded as $t$ approaches 1. To prove Theorem 3.1 we need a lemma which appears as Lemma 1.3 in [DPS1]. For the reader's convenience, we include a proof here.

Lemma 3.1. In the above situation there exists a constant $C_{1}$ that depends only on $n, c_{1}, \omega_{0}$, such that given any $\delta>0$ there exists an open set $U_{\delta} \subset X$ such that its diameter with respect to $\omega$ is less than $C_{1} \delta^{-1 / 2}$ and its volume with respect to $\omega_{0}$ is at least $\int_{X} \omega_{0}^{n}-\delta$. 
Proof. First notice that 3.2 gives a uniform $L^{1}$ bound on $\omega$. Up to covering $X$ by finitely many charts, we may assume that $X=K$ is a compact convex set in $\mathbb{C}^{n}$, and we will denote by $g_{E}$ the Euclidean metric on $K$. If $x_{1}, x_{2} \in K$, we denote by $\left[x_{1}, x_{2}\right]$ the segment joining them in $K$, and we compute the average of the length square of $\left[x_{1}, x_{2}\right]$ with respect to $\omega$, when the endpoints vary. Using Fubini's Theorem and the Cauchy-Schwarz inequality we get

$$
\begin{aligned}
& \int_{K \times K}\left(\int_{0}^{1} \sqrt{\omega\left((1-s) x_{1}+s x_{2}\right)\left(x_{2}-x_{1}\right)} d s\right)^{2} d x_{1} d x_{2} \\
& \leq\left\|x_{2}-x_{1}\right\|_{g_{E}}^{2} \int_{0}^{1} \int_{K \times K}\left|\omega\left((1-s) x_{1}+s x_{2}\right)\right| d x_{1} d x_{2} d s \\
& \leq \operatorname{diam}_{g_{E}}^{2}(K) 2^{2 n}\left(\int_{0}^{1 / 2} \int_{K \times K}\left|\omega\left(y+s x_{2}\right)\right| d y d x_{2} d s\right. \\
&\left.+\int_{1 / 2}^{1} \int_{K \times K}\left|\omega\left((1-s) x_{1}+y\right)\right| d y d x_{1} d s\right) \\
& \leq \operatorname{diam}_{g_{E}}^{2}(K) 2^{2 n} \operatorname{Vol}_{g_{E}}(K)\|\omega\|_{L^{1}(K)} \leq C_{1},
\end{aligned}
$$

where $C_{1}$ is a uniform constant; we have changed variable $y=(1-s) x_{1}$ if $s \leq 1 / 2$ and $y=s x_{2}$ when $s \geq 1 / 2$ and integrated first with respect to $y$. Then the set $S$ of pairs $\left(x_{1}, x_{2}\right) \in K \times K$ such that the length of $\left[x_{1}, x_{2}\right]$ with respect to $\omega$ is more than $\left(C_{1} / \delta\right)^{1 / 2}$ has Euclidean measure less than or equal to $\delta$ : otherwise

$$
\begin{aligned}
& \int_{K \times K}\left(\int_{0}^{1} \sqrt{\omega\left((1-s) x_{1}+s x_{2}\right)\left(x_{2}-x_{1}\right)} d s\right)^{2} d x_{1} d x_{2} \\
& \quad \geq \int_{S}\left(\int_{0}^{1} \sqrt{\omega\left((1-s) x_{1}+s x_{2}\right)\left(x_{2}-x_{1}\right)} d s\right)^{2} d x_{1} d x_{2} \geq \frac{C_{1}}{\delta} \operatorname{Vol}_{g_{E}}(S),
\end{aligned}
$$

which is more than $C_{1}$, and this contradicts 3.3 . If $x_{1} \in K$ we let $S\left(x_{1}\right)$ be the set of all $x_{2} \in K$ such that $\left(x_{1}, x_{2}\right) \in S$; moreover, we let $Q$ be the set of $x_{1} \in K$ such that $\operatorname{Vol}_{g_{E}}\left(S\left(x_{1}\right)\right) \geq \frac{1}{2} \operatorname{Vol}_{g_{E}}(K)$ and $R$ be the set of $\left(x_{1}, x_{2}\right) \in S$ such that $x_{1} \in Q$. Then by Fubini's Theorem,

$$
\delta \geq \operatorname{Vol}_{g_{E}}(R)=\int_{R} d x_{2} d x_{1}=\int_{Q}\left(\int_{S\left(x_{1}\right)} d x_{2}\right) d x_{1} \geq \frac{1}{2} \operatorname{Vol}_{g_{E}}(K) \operatorname{Vol}_{g_{E}}(Q),
$$

and so $\operatorname{Vol}_{g_{E}}(Q) \leq 2 \delta / \operatorname{Vol}_{g_{E}}(K)$. We let $U_{\delta}=K \backslash Q$. Then $U_{\delta}$ is open and if $x_{1}, x_{2} \in U_{\delta}$ then $\operatorname{Vol}_{g_{E}}\left(S\left(x_{i}\right)\right)<\frac{1}{2} \operatorname{Vol}_{g_{E}}(K)$ for $i=1,2$. Hence $\operatorname{Vol}_{g_{E}}\left(\left(K \backslash S\left(x_{1}\right)\right) \cap\left(K \backslash S\left(x_{2}\right)\right)\right)>0$ and so this set is nonempty. If $y$ belongs to it, then $\left(x_{1}, y\right)$ and $\left(x_{2}, y\right)$ are not in $S$, which means that the lengths with respect to $\omega$ of the segments $\left[x_{1}, y\right]$ and $\left[y, x_{2}\right]$ are both less than $\left(C_{1} / \delta\right)^{1 / 2}$. Concatenating these two segments we get a path from $x_{1}$ to $x_{2}$ with length less than $2\left(C_{1} / \delta\right)^{1 / 2}$. We also have

$$
\operatorname{Vol}_{\omega_{0}}(Q) \leq C_{2} \operatorname{Vol}_{g_{E}}(Q) \leq \frac{2 C_{2} \delta}{\operatorname{Vol}_{g_{E}}(K)} .
$$

Up to adjusting the constants, this is what we want. 
Proof of Theorem 3.1. Choose $\delta \leq \min \left(C_{1}^{2}, \frac{1}{2} \int_{X} \omega_{0}^{n}\right)$, and pick any $p \in U_{\delta}$. If we denote the metric ball of $\omega$ centered at $p$ and with radius $r$ by $B(p, r)$, then $U_{\delta} \subset B\left(p, C_{2}\right)$, where $C_{2}=C_{1} \delta^{-1 / 2} \geq 1$. Hence

$$
\int_{B\left(p, C_{2}\right)} \omega_{0}^{n} \geq \int_{U_{\delta}} \omega_{0}^{n} \geq \frac{1}{2} \int_{X} \omega_{0}^{n} .
$$

Proceeding as in (3.1) we see that $\omega^{n}=B \omega_{0}^{n}$, where

$$
B=\frac{\int_{X} \omega^{n}}{\int_{X} \omega_{0}^{n}}
$$

So we get

$$
\int_{B\left(p, C_{2}\right)} \omega^{n} \geq B C_{3}
$$

for some constant $C_{3}>0$ independent of $\omega$. Since $\operatorname{Ric}(\omega)=0$, the Bishop volume comparison theorem and (3.5) give

$$
\int_{B(p, 1)} \omega^{n} \geq \frac{\int_{B\left(p, C_{2}\right)} \omega^{n}}{C_{2}^{2 n}} \geq B C_{4}>0
$$

The following lemma is due to Yau (see e.g. Theorem I.4.1 in [SY]), but we provide a proof for completeness.

Lemma 3.2. Let $\left(M^{2 n}, g\right)$ be a closed Riemannian manifold with $\operatorname{Ric}(g) \geq 0$, let $p \in M$ and $1<R<\operatorname{diam}(X, g)$. Then

$$
\frac{R-1}{4 n} \leq \frac{\operatorname{Vol}(B(p, 2(R+1)))}{\operatorname{Vol}(B(p, 1))} .
$$

Proof. Choose $x_{0} \in \partial B(p, R)$, so that $d\left(x_{0}, p\right)=R$, and define $\rho(x)=d\left(x, x_{0}\right)$. The Laplacian comparison theorem gives $\Delta \rho^{2} \leq 4 n$ in the sense of distributions. Let $\varphi(x)=\psi(\rho(x))$ where

$$
\psi(t)= \begin{cases}1 & \text { if } 0 \leq t \leq R-1 \\ \frac{1}{2}(R+1-t) & \text { if } R-1<t<R+1, \\ 0 & \text { if } t \geq R+1\end{cases}
$$

Then $\varphi$ is a nonnegative Lipschitz function supported in $B\left(x_{0}, R+1\right)$, and we have

$$
\begin{aligned}
\int_{M} \varphi \Delta \rho^{2} d V_{g} & =-\int_{B\left(x_{0}, R+1\right)} \nabla \varphi \cdot \nabla \rho^{2} d V_{g}=-2 \int_{B\left(x_{0}, R+1\right)} \rho|\nabla \rho|^{2} \psi^{\prime}(\rho(x)) d V_{g} \\
& =\int_{B\left(x_{0}, R+1\right) \backslash B\left(x_{0}, R-1\right)} \rho d V_{g} \\
& \geq(R-1) \operatorname{Vol}\left(B\left(x_{0}, R+1\right) \backslash B\left(x_{0}, R-1\right)\right),
\end{aligned}
$$


and also

$$
\int_{M} \varphi \Delta \rho^{2} d V_{g} \leq 4 n \int_{B\left(x_{0}, R+1\right)} \varphi d V_{g} \leq 4 n \operatorname{Vol}\left(B\left(x_{0}, R+1\right)\right) .
$$

Notice that $B(p, 1) \subset B\left(x_{0}, R+1\right) \backslash B\left(x_{0}, R-1\right)$ and so the previous two estimates give

$$
(R-1) \operatorname{Vol}(B(p, 1)) \leq 4 n \operatorname{Vol}\left(B\left(x_{0}, R+1\right)\right) .
$$

The conclusion follows from the fact that $B\left(x_{0}, R+1\right) \subset B(p, 2(R+1))$.

Lemma 3.2 shows that for any $1<R<\operatorname{diam}(X, \omega)$ we have

$$
\frac{R-1}{4 n} \leq \frac{\int_{B(p, 2(R+1))} \omega^{n}}{\int_{B(p, 1)} \omega^{n}} .
$$

Choosing $R=\operatorname{diam}(X, \omega)-1$ and using (3.6), 3.4 we get

$$
\operatorname{diam}(X, \omega) \leq 2+\frac{4 n}{B C_{4}} \int_{X} \omega^{n}=2+\frac{4 n}{C_{4}} \int_{X} \omega_{0}^{n},
$$

which is bounded independently of $\omega$. This completes the proof of Theorem 3.1 (a somewhat similar argument can be found in [[P1]).

Once we have the diameter bound, we can apply the Bishop volume comparison theorem again to deduce that for any point $p \in X$ and any $r>0, t<1$,

$$
\int_{B_{t}(p, r)} \omega_{t}^{n} \geq r^{2 n} \frac{\int_{X} \omega_{t}^{n}}{\operatorname{diam}\left(X, \omega_{t}\right)^{2 n}} \geq c r^{2 n},
$$

where $c>0$ is a uniform constant. A well-known computation in Chern-Weil theory gives

$$
\frac{1}{n(n-1)} \int_{X}\left\|\operatorname{Rm}_{t}\right\|_{t}^{2} \omega_{t}^{n}=\int_{X} c_{2}\left(X, \omega_{t}\right) \wedge \omega_{t}^{n-2}=c_{2}(X) \cdot \alpha_{t}^{n-2} \leq C,
$$

where $\mathrm{Rm}_{t}$ is the Riemann curvature tensor of $\omega_{t}$ and $c_{2}\left(X, \omega_{t}\right)$ is the second Chern form of $\omega_{t}$. If $n=2$ we can thus apply Theorem $\mathrm{C}$ of [An], Theorem 5.5 of [BKN] or Proposition 3.2 of [Ti] to infer that a subsequence of $\left(X, \omega_{t}\right)$ converges to an Einstein orbifold with isolated singularities in the Gromov-Hausdorff topology, and also in the $C^{\infty}$ topology on compact sets outside the orbifold points. If $n>2$ these theorems require a uniform bound on

$$
\int_{X}\left\|\mathrm{Rm}_{t}\right\|_{t}^{n} \omega_{t}^{n}
$$

which in general cannot be expressed in terms of topological data as above. Instead when $n>2$ we apply a general theorem of Gromov $[\mathrm{Gr}]$ that says that any sequence of compact Riemannian manifolds of dimension $2 n$ with diameter bounded above and Ricci curvature bounded below, has a subsequence that converges in the Gromov-Hausdorff topology to a compact length space. Thus a subsequence of $\left(X, \omega_{t}\right)$ converges to a compact metric 
space $Y$, and Theorem 1.15 in [CCT] says that $Y$ is a complex manifold outside a rectifiable set $R \subset Y$ of real Hausdorff codimension at least 4. Moreover, their Theorem 9.1 gives supporting evidence that $R$ should in fact be a complex subvariety of $Y$.

On the other hand, our Theorem 1.1 gives the convergence of the whole sequence of metrics, and not just of a subsequence, and the limit metric is uniquely determined by the class $\alpha$. When $n>2$ the convergence we get is stronger than Gromov-Hausdorff convergence, but it only holds outside the singular set $E$. Also we see precisely what the limit space $Y$ is, namely the Calabi-Yau model of $X$ obtained from the contraction map of $\alpha$. It has canonical singularities, so its singular set is a subvariety of complex codimension at least 2 , and when $n=2$ canonical singularities are precisely rational double points, which are of orbifold type. We will discuss the case $n=2$ in more detail in Section 5 Let us also mention the results of Ruan [Ru]. He studies the Gromov-Hausdorff limits of sequences of Kähler metrics on a fixed compact manifold $X$, with uniformly bounded sectional curvature. Roughly speaking, he proves that there exists an analytic subvariety $E \subset X$ such that a subsequence of the metrics converges in the Gromov-Hausdorff topology on $X \backslash E$ to a smooth Hermitian form $\omega$, which is either Kähler (noncollapsing) or pointwise nonnegative with determinant zero (collapsing). Moreover, in the collapsing case, the kernel of $\omega$ gives a holomorphic foliation with singularities on $X$. Unfortunately in our setting the curvature is not bounded in general, so Ruan's results do not apply, but our Theorem 1.1 gives in particular Ruan's conclusion in the noncollapsing case. We will discuss the collapsing case in Section 6 .

Of course, the above-mentioned results apply in more general situations than ours. Also, all the results in this section work in the case when $X$ is not projective, and the ample cone is replaced by the (bigger) Kähler cone. Then the above theorems still apply, but for technical reasons our Theorem 1.1 does not (see Section 6 for more discussions).

\section{Limits of Ricci-flat metrics}

In this section we will prove Theorem 1.1 The idea is to carefully set up a family of complex Monge-Ampère equations that degenerate in the limit, and prove estimates for the solutions that are uniform outside a subvariety.

We begin with

Proposition 4.1. Let $X$ be a projective Calabi-Yau $n$-fold, and $\alpha \in N^{1}(X)_{\mathbb{R}}$ a big and nef class that is not ample. Then there exists a smooth real $(1,1)$ form $\omega \in \alpha$ that is pointwise nonnegative and which is Kähler outside a proper analytic subvariety of $X$. Moreover, if $\alpha_{t}:[0,1] \rightarrow \overline{\mathcal{K}}_{N S}$ is a smooth path such that $\alpha_{t} \in \mathcal{K}_{N S}$ for $t<1$ and $\alpha_{1}=\alpha$, then we can find a continuous family of Kähler forms $\beta_{t} \in \alpha_{t}, t<1$, such that $\beta_{t} \rightarrow \omega$ in the $C^{\infty}$ topology as $t$ approaches 1 .

Proof. Let us assume first that that $\alpha=c_{1}(L)$ for some line bundle $L$, which is equivalent to requiring that $\alpha \in N^{1}(X)_{\mathbb{Z}}$. Now $L$ is nef and big and so Theorem 2.1 implies that $L$ is semiample, so there exists some $k \geq 1$ such that $k L$ is globally generated. This gives 
a morphism $f: X \rightarrow \mathbb{P}^{N}$ such that $f^{*} \mathcal{O}(1)=k L$. If we let $\omega_{F S}$ be the Fubini-Study metric on $\mathbb{P}^{N}$, then $\omega=f^{*} \omega_{F S} / k$ is a pointwise nonnegative smooth real $(1,1)$ form in the class $\alpha$. Moreover, $\omega$ is Kähler outside the exceptional set of $f$, which is a proper subvariety of $X$. If $\alpha \in N^{1}(X)_{\mathbb{Q}}$, then $k \alpha \in N^{1}(X)_{\mathbb{Z}}$ for some integer $k \geq 1$, and we can proceed as above. If finally $\alpha \in N^{1}(X)_{\mathbb{R}}$ then by Theorem 2.4 we know that the subcone of nef and big classes is locally rational polyhedral. Hence $\alpha$ lies on a face of this cone which is cut out by linear equations with rational coefficients. It follows that rational points on this face are dense, and it is then possible to write $\alpha$ as a linear combination of classes in $N^{1}(X)_{\mathbb{Q}}$ which are nef and big, with nonnegative coefficients. It is now clear that we can represent $\alpha$ by a smooth nonnegative form $\omega$. Notice that all of these classes give the same contraction map $f: X \rightarrow Y$, because they lie on the same face. This map is then also the contraction map of $\alpha$, and $\omega$ is again Kähler outside the exceptional set of $f$.

Now fix a ball $\mathcal{U}$ in $N^{1}(X)_{\mathbb{R}}$ centered at $\alpha$ such that $\mathcal{K}_{N S} \cap \mathcal{U}$ is defined by the inequalities $\left\{\Phi_{\beta}>0\right\}_{1 \leq \beta \leq k}$ where the $\Phi_{\beta}$ are linear forms with rational coefficients. Since the big cone is open, up to shrinking $\mathcal{U}$ we may also assume that all the classes in $\partial \mathcal{K}_{N S} \cap \mathcal{U}$ are big. We may add some more linear forms to the $\Phi_{\beta}$, until they define a strongly convex rational polyhedral cone $C$ which is contained in $\overline{\mathcal{K}}_{N S} \cap \mathcal{U}$. We can then write

$$
C=\left\{\sum_{i=1}^{\ell} a_{i} \gamma_{i} \mid a_{i} \geq 0\right\},
$$

where the $\gamma_{i}$ are nef and big classes in $\mathcal{U}$. We claim that, when $t$ is greater than some $t_{0}<1$, it is possible to write the path $\alpha_{t}$ as $\sum_{i} a_{i}(t) \gamma_{i}$ where the functions $a_{i}(t)$ are continuous and nonnegative. Assume first that the cone $C$ is simplicial, which means that the $\gamma_{i}$ are linearly independent. Then the path $\alpha_{t}$ enters and eventually stays in $C$, and so it can be expressed uniquely as

$$
\alpha_{t}=\sum_{i=1}^{\ell} a_{i}(t) \gamma_{i},
$$

where the $a_{i}(t)$ are smooth and nonnegative, $t_{0} \leq t \leq 1$. If on the other hand $C$ is not simplicial, it can be written as a finite union of simplicial subcones that intersect only along faces, and are spanned by some linearly independent subsets of the $\gamma_{i}$. On any time interval when $\alpha_{t}$ belongs to the interior of a simplicial cone, the coefficients $a_{i}(t)$ in (4.1) vary smoothly, and on a common face of two simplicial cones the coefficients agree, hence the $a_{i}(t)$ vary continuously when $t_{0} \leq t<1$. Moreover, since we only have finitely many simplicial subcones, we see that as $t \rightarrow 1$ the $a_{i}(t)$ converge to the coefficients of $\alpha_{1}$ in any of the simplicial cones that contain it, and so the $a_{i}(t)$ are continuous on the whole interval $t_{0} \leq t \leq 1$.

By the first part of the proof we know that we can choose a smooth nonnegative representative $\delta_{i} \in \gamma_{i}$, for all $i$. Choose a smooth function $\varepsilon:\left[t_{0}, 1\right] \rightarrow \mathbb{R}$ that is positive on $\left[t_{0}, 1\right)$ and $\varepsilon(1)=0$, and that is small enough so that the classes $\tilde{\alpha}_{t}=\alpha_{t}-\varepsilon(t) \alpha_{t_{0}}$ are ample for all $t_{0} \leq t<1$. Then the new path $\tilde{\alpha}_{t}$ is also converging to $\alpha$ as $t \rightarrow 1$, and by 
the previous claim we can write

$$
\tilde{\alpha}_{t}=\sum_{i=1}^{\ell} \tilde{a}_{i}(t) \gamma_{i},
$$

where $\tilde{a}_{i}(t)$ is a continuous nonnegative function for all $i$. Then the smooth $(1,1)$ forms

$$
\tilde{\beta}_{t}:=\sum_{i=1}^{\ell} \tilde{a}_{i}(t) \delta_{i}
$$

are nonnegative representatives of $\tilde{\alpha}_{t}$ that vary continuously in $t$. When $t$ approaches 1 , the forms $\tilde{\beta}_{t}$ converge in the $C^{\infty}$ topology to a smooth nonnegative form $\tilde{\omega}$ representing $\alpha$. If $\chi$ is a Kähler form in $\alpha_{t_{0}}$, then the forms $\beta_{t}=\tilde{\beta}_{t}+\varepsilon(t) \chi$ defined on [t $\left.t_{0}, 1\right)$ are Kähler, represent $\alpha_{t}$ and converge to $\tilde{\omega}$ as $t \rightarrow 1$. Up to replacing $\omega$ by $\tilde{\omega}$, this gives the desired family of forms on $\left[t_{0}, 1\right)$. It is very easy to extend the family $\beta_{t}$ on the whole $[0,1)$, and since we are not going to use this, we leave the proof to the reader.

Of course, a similar statement holds if we are given a sequence of ample classes $\alpha_{i}$ converging to $\alpha$, instead of a path.

Let us now recall some notation and facts from analytic geometry. If $X$ is any complex manifold and $\omega$ is a Hermitian form on $X$, we will denote by $P S H(X, \omega)$ the set of all upper semicontinuous (usc) functions $\varphi: X \rightarrow[-\infty,+\infty)$ such that $\omega+\sqrt{-1} \partial \bar{\partial} \varphi$ is a positive current. If $(X, \omega)$ is Kähler, then all Kähler potentials for $\omega$ belong to $\operatorname{PSH}(X, \omega)$. A fundamental result by Bedford-Taylor [BT] says that the Monge-Ampère operator $(\omega+\sqrt{-1} \partial \bar{\partial} \varphi)^{n}$ is well defined whenever $\varphi \in P S H(X, \omega)$ is locally bounded. Let us also recall the definition of a singular Kähler metric [EGZ] on a (possibly singular) algebraic variety $X$. This is given by specifying its Kähler potentials on an open cover $\left(U_{i}\right)$ of $X$, which are usc functions $\varphi_{i}: U_{i} \rightarrow[-\infty,+\infty)$ with the following property: $\varphi_{i}$ extends to a plurisubharmonic function on an open set $V_{i} \subset \mathbb{C}^{m}$ where $U_{i} \subset V_{i}$ is a local embedding. We refer the reader to Section 7 of [EGZ] for the definition of a singular Ricci-flat Kähler metric and for a proof that they always exist on Calabi-Yau models. With these facts in mind, we can now give the

Proof of Theorem 1.1. Proposition 4.1 gives us a smooth nonnegative representative $\omega \in \alpha$, and continuously varying Kähler forms $\beta_{t} \in \alpha_{t}$, for $t<1$, such that $\beta_{t} \rightarrow \omega$ as $t \rightarrow 1$. As in the proof of Proposition 4.1, there is a contraction map $f: X \rightarrow Y$ such that $Y$ is a normal irreducible projective variety, $f$ is birational and $f_{*} \mathcal{O}_{X}=\mathcal{O}_{Y}$. Moreover, $\omega$ is the pullback of a (singular) Kähler metric on $Y$, and it is Kähler outside the exceptional set of $f$. Then setting $D_{0}=0$ as Cartier divisors on $Y$, we have $a K_{X}=f^{*} D_{0}$ for some integer $a>0$, so

$$
f_{*}\left(a K_{X}\right)=D_{0}=0
$$

holds as Weil divisors, but since $f$ is birational we also have $f_{*}\left(a K_{X}\right)=a K_{Y}$ (as Weil divisors), hence $a K_{Y}$ is Cartier and is equal to zero. So we have $f^{*} K_{Y}=K_{X}$ as $\mathbb{Q}$ divisors, which implies that $Y$ has at most canonical singularities and is a Calabi-Yau model (see also Corollary 1.5 of [ [Ka1] $)$. 
Denote by $\Omega$ the smooth volume form on $X$ given by

$$
\Omega=\frac{\omega_{0}^{n}}{\int_{X} \omega_{0}^{n}},
$$

which satisfies $\int_{X} \Omega=1$. We can write $\Omega=F \omega^{n}$, where $F \in L^{1}\left(\omega^{n}\right), F>0$. The following argument to show that actually $F \in L^{p}\left(\omega^{n}\right)$ for some $p>1$ is similar to Lemma 3.2 in [EGZ]. First of all, $1 / F$ is smooth, nonnegative, and vanishes precisely on the exceptional set of $f$. Fixing local coordinates $\left(z^{i}\right)$ on a polydisc $D \subset X$ and a local embedding $G: f(D) \rightarrow \mathbb{C}^{m}$, we see that $1 / F$ is comparable to

$$
\left|\frac{\partial G}{\partial z^{1}} \wedge \cdots \wedge \frac{\partial G}{\partial z^{n}}\right|^{2}
$$

on $D$. But this is in turn comparable to

$$
\sum_{i=1}^{r}\left|g_{i}\right|^{2}
$$

where the $g_{i}$ are holomorphic functions on $D$, and so $F^{\varepsilon} \in L^{1}(D, \Omega)$ for some small $\varepsilon>0$ that depends on the vanishing orders of the $g_{i}$. Then

$$
\int_{D} F^{1+\varepsilon} \omega^{n}=\int_{D} F^{\varepsilon} \Omega<\infty .
$$

The compactness of $X$ gives $F \in L^{1+\varepsilon}\left(\omega^{n}\right)$, and so we can apply Theorem 2.1 and Proposition 3.1 of [EGZ] (which rely on the seminal work of Kołodziej [Koł]) to get a unique bounded $\varphi \in P S H(X, \omega)$ such that

$$
(\omega+\sqrt{-1} \partial \bar{\partial} \varphi)^{n}=\alpha^{n} \Omega,
$$

and $\sup _{X} \varphi=0$. We then embed $Y$ into projective space and extend $\omega$ to a Kähler form in a neighborhood of $Y$ as in Proposition 3.3 of [DP]. Composing the embedding with $f$ we get a morphism which is birational with the image, with connected fibers, and we can then apply Theorem 1.1 in [Z1] (see also [Z2] and Remark 5.2 in [DZ]) to conclude that $\varphi$ is continuous. Moreover, we can see that $\varphi$ descends to a function on $Y$ : if $V$ is a fiber of $f$, the restriction of $\varphi$ to $V$ is a plurisubharmonic function, because $\left.\omega\right|_{V}=0$. Desingularizing $V$ and applying the maximum principle we see that $\left.\varphi\right|_{V}$ has to be constant, and so $\varphi$ descends to $Y$. Since $\omega$ by construction is the pullback of a (singular) Kähler form on $Y$, we see that $\omega+\sqrt{-1} \partial \bar{\partial} \varphi$ is a singular Ricci-flat metric on $Y$, in the terminology of [EGZ]. On $X$, the closed positive current $\omega_{1}=\omega+\sqrt{-1} \partial \bar{\partial} \varphi$ clearly lies in the class $\alpha$ and has continuous potentials.

Intuitively, our goal is to get estimates in the open set where $\omega$ is positive. This can be done rigorously in the following way, which was first used by H. Tsuji [Ts] (see also [TZ], [CL] for a recent revisiting of his approach). Since $\alpha$ is nef and big, by Kodaira's lemma (Example 2.2.23 in [L]) there exists an effective Cartier $\mathbb{R}$-divisor $E$ such that for all $\varepsilon>0$ small enough, $\alpha-\varepsilon E=\kappa_{\varepsilon}$ is Kähler. 
We will show that $\varphi$ is smooth on $X \backslash E$, and so $\omega_{1}$ is a smooth Ricci-flat metric there, and that the Ricci-flat metrics $\omega_{t}$ converge to $\omega_{1}$ in the $C^{\infty}$ topology on compact sets of $X \backslash E$. Notice that the metric $\omega_{1}$ on $X \backslash E$ cannot be complete, since its diameter is finite by Theorem 3.1 . Once this is proved, we can repeat the argument for any other $E$ given by Kodaira's lemma, and by uniqueness we see that $\omega_{1}$ is smooth off $E^{\prime}$, the intersection of the supports of all such $E$. We claim that if $\alpha=c_{1}(L)$ for some line bundle $L$, then $E^{\prime}$ is equal to the null locus of $L$. By Nakamaye's Theorem all we need to show is that it is equal to the augmented base locus of $L$. If $x \in X$ is a point outside the augmented base locus, then there exist an ample divisor $H$ and $k, m$ large enough so that $x$ is not in the base locus of $m L-(m / k) H$. But this means that $m L-(m / k) H \sim N$ where $N$ is an effective divisor that does not pass through $x$, and moreover the cohomology class of $L-(1 / m) N$ is Kähler. So we can take $\varepsilon=1 / m$ and $E=N$, and we see that $E^{\prime}$ is contained in the null locus of $L$. Conversely, if $x$ belongs to the null locus, then there exists a subvariety $V$ through $x$ with $\operatorname{dim} V=k$ and $\left(L^{k} \cdot V\right)=0$. Since the potentials for the current $\omega_{1}$ are continuous, the self-intersection $\omega_{1}^{k}$ is a welldefined closed positive current [BT], which restricts to a nonnegative Borel measure on $V$. The integral $\int_{V} \omega_{1}^{k}$ is then equal to the cohomological intersection number $\left(L^{k} \cdot V\right)$ (see e.g. Corollary 9.3 in $[\mathrm{D}]$ ), which is zero. But if $x$ is not in $E^{\prime}$ then $\omega_{1}$ is smooth and Kähler near $x$ and the volume of $V$ with respect to $\omega_{1}$ would be positive, which is a contradiction.

Fix once and for all an $\varepsilon>0$ small enough so that the conclusion of Kodaira's lemma holds. First of all notice that the classes $\alpha_{t}-\varepsilon E=\kappa_{\varepsilon}^{t}$ are all Kähler when $t$ is close to 1 . Choose a Kähler form $\chi_{\varepsilon} \in \kappa_{\varepsilon}$, let $\sigma \in H^{0}\left(X, \mathcal{O}_{X}(E)\right)$ be the canonical section, and fix a Hermitian metric $|\cdot|$ on $E$ such that the following Poincaré-Lelong equation holds:

$$
\omega-\varepsilon[E]=\chi_{\varepsilon}-\varepsilon \sqrt{-1} \partial \bar{\partial} \log |\sigma|
$$

where $[E]$ denotes the current of integration on $E$. Then we have

$$
\beta_{t}-\varepsilon[E]=\chi_{\varepsilon}+\left(\beta_{t}-\omega\right)-\varepsilon \sqrt{-1} \partial \bar{\partial} \log |\sigma|
$$

and $\chi_{\varepsilon}^{t}=\chi_{\varepsilon}+\left(\beta_{t}-\omega\right)$ is Kähler for $t$ close to 1 . There are smooth functions $\varphi_{t}$ solving

$$
\omega_{t}^{n}=\left(\beta_{t}+\sqrt{-1} \partial \bar{\partial} \varphi_{t}\right)^{n}=\alpha_{t}^{n} \Omega
$$

where the positive constants $\alpha_{t}^{n}$ approach $\alpha^{n}$ as $t$ goes to 1 , and $\sup _{X} \varphi_{t}=0$. We now derive a uniform $L^{\infty}$ estimate for $\varphi_{t}$. Since the Ricci-flat metrics $\omega_{t}$ have a uniform upper bound on the diameter by Theorem 3.1 and a uniform positive lower bound for the volume $\int_{X} \omega_{t}^{n}$, classical results of Croke [Cr], Li [Li] and Li-Yau [LY] give uniform upper bounds for the Sobolev and Poincaré constants of $\omega_{t}$. We temporarily modify the normalization of $\varphi_{t}$ by requiring that $\int_{X} \varphi_{t} \omega_{t}^{n}=0$ and we are going to show that $\left|\varphi_{t}\right| \leq C$. This will then hold for the original $\varphi_{t}$ as well, with perhaps a bigger constant. We employ a Moser iteration argument in the following way, inspired by [Y2]. For any $p>1$ we 
compute

$$
\begin{aligned}
\int_{X}\left|\nabla\left(\varphi_{t}\left|\varphi_{t}\right|^{(p-2) / 2}\right)\right|_{\omega_{t}}^{2} \omega_{t}^{n} & =\frac{p^{2}}{4} \int_{X}\left|\varphi_{t}\right|^{p-2}\left|\nabla \varphi_{t}\right|_{\omega_{t}}^{2} \omega_{t}^{n} \\
& =\frac{n p^{2}}{4} \int_{X}\left|\varphi_{t}\right|^{p-2} \partial \varphi_{t} \wedge \bar{\partial} \varphi_{t} \wedge \omega_{t}^{n-1} \\
& \leq \frac{n p^{2}}{4} \int_{X}\left|\varphi_{t}\right|^{p-2} \partial \varphi_{t} \wedge \bar{\partial} \varphi_{t} \wedge\left(\sum_{i=0}^{n-1} \omega_{t}^{n-1-i} \wedge \beta_{t}^{i}\right) \\
& =-\frac{n p^{2}}{4(p-1)} \int_{X} \varphi_{t}\left|\varphi_{t}\right|^{p-2} \partial \bar{\partial} \varphi_{t} \wedge\left(\sum_{i=0}^{n-1} \omega_{t}^{n-1-i} \wedge \beta_{t}^{i}\right) \\
& =\frac{n p^{2}}{4(p-1)} \int_{X} \varphi_{t}\left|\varphi_{t}\right|^{p-2}\left(\beta_{t}-\omega_{t}\right) \wedge\left(\sum_{i=0}^{n-1} \omega_{t}^{n-1-i} \wedge \beta_{t}^{i}\right) \\
& =\frac{n p^{2}}{4(p-1)} \int_{X} \varphi_{t}\left|\varphi_{t}\right|^{p-2}\left(\beta_{t}^{n}-\omega_{t}^{n}\right) \\
& \leq C p \int_{X}\left|\varphi_{t}\right|^{p-1} \omega_{t}^{n},
\end{aligned}
$$

where we used 4.5) in the last inequality. Using 4.6 and the uniform Sobolev inequality for $\omega_{t}$ and iterating in a standard way (see e.g. [Si2]) we get

$$
\left\|\varphi_{t}\right\|_{L^{\infty}} \leq C\left(\int_{X}\left|\varphi_{t}\right|^{2} \omega_{t}^{n}\right)^{1 / 2}
$$

We then use the uniform Poincare inequality for $\omega_{t}$ together with 4.6 with $p=2$ and with the Hölder inequality and the fact that the volume $\int_{X} \omega_{t}^{n}$ is bounded above to get

$$
\int_{X}\left|\varphi_{t}\right|^{2} \omega_{t}^{n} \leq C \int_{X}\left|\nabla \varphi_{t}\right|_{\omega_{t}}^{2} \omega_{t}^{n} \leq C \int_{X}\left|\varphi_{t}\right| \omega_{t}^{n} \leq C\left(\int_{X}\left|\varphi_{t}\right|^{2} \omega_{t}^{n}\right)^{1 / 2}
$$

which gives $\int_{X}\left|\varphi_{t}\right|^{2} \omega_{t}^{n} \leq C$, and so with 4.7) this completes the proof of the $L^{\infty}$ bound $\left|\varphi_{t}\right| \leq C$. Notice that such a bound also follows from [EGZ], but our proof is more elementary.

Outside $E$ we have

$$
\beta_{t}=\chi_{\varepsilon}^{t}-\varepsilon \sqrt{-1} \partial \bar{\partial} \log |\sigma|,
$$

so that the functions $\psi_{t}=\varphi_{t}-\varepsilon \log |\sigma|$ solve

$$
\left(\chi_{\varepsilon}^{t}+\sqrt{-1} \partial \bar{\partial} \psi_{t}\right)^{n}=\alpha_{t}^{n} \Omega=e^{F_{\varepsilon}^{t}}\left(\chi_{\varepsilon}^{t}\right)^{n}
$$

there, for some appropriate smooth functions $F_{\varepsilon}^{t}$, defined on the whole of $X$. As $t$ approaches 1 , the Kähler forms $\chi_{\varepsilon}^{t}$ are uniformly bounded in the smooth topology (with 
eigenvalues bounded away from 0 uniformly), and so are the functions $F_{\varepsilon}^{t}$. Yau's second order estimates [Y2] for the Monge-Ampère equation (4.8) give

$$
\triangle_{t}^{\prime}\left(e^{-A \psi_{t}}\left(n+\triangle_{t} \psi_{t}\right)\right) \geq e^{-A \psi_{t}}\left(-C_{1}-C_{2}\left(n+\triangle_{t} \psi_{t}\right)+\left(n+\triangle_{t} \psi_{t}\right)^{n /(n-1)}\right),
$$

where $A, C_{1}$ and $C_{2}$ are uniform positive constants, $\triangle_{t}$ is the Laplacian of $\chi_{\varepsilon}^{t}$, and $\triangle_{t}^{\prime}$ is the Laplacian of $\chi_{\varepsilon}^{t}+\sqrt{-1} \partial \bar{\partial} \psi_{t}$. Now notice that on $X \backslash E$ we have

$$
e^{-A \psi_{t}}\left(n+\triangle_{t} \psi_{t}\right)=|\sigma|^{A \varepsilon} e^{-A \varphi_{t}}\left(n+\triangle_{t} \varphi_{t}-\varepsilon \triangle_{t} \log |\sigma|\right),
$$

and

$$
\left|\triangle_{t} \log \right| \sigma|| \leq C,
$$

for some uniform constant $C$. Hence the function $e^{-A \psi_{t}}\left(n+\triangle_{t} \psi_{t}\right)$ goes to zero when we approach $E$, and so its maximum will be attained. The maximum principle applied to (4.9) then gives

$$
n+\triangle_{t} \psi_{t} \leq C e^{A\left(\psi_{t}-\inf _{X \backslash E} \psi_{t}\right)}
$$

on the whole of $X \backslash E$. But noticing that $\inf _{X \backslash E} \psi_{t} \geq \inf _{X} \varphi_{t}-C$ for a uniform constant $C$, and recalling that $\left|\varphi_{t}\right| \leq C_{0}$, we get

$$
n+\triangle_{t} \varphi_{t} \leq C+n+\triangle_{t} \psi_{t} \leq C\left(1+|\sigma|^{-A \varepsilon}\right) .
$$

This gives uniform interior $C^{2}$ estimates of $\varphi_{t}$ and $\psi_{t}$ on compact subsets of $X \backslash E$. Then the Harnack estimate of Evans-Krylov gives uniform $C^{2, \gamma}$ estimates, for some $0<\gamma<1$, and a standard bootstrapping argument gives uniform $C^{k, \gamma}$ estimates for all $k \geq 2$, on compact sets of $X \backslash E$. Thus the family $\left(\varphi_{t}\right)$ is precompact $C^{k, \gamma^{\prime}}(X \backslash E)$ for any $0<\gamma^{\prime}<\gamma$, and any limit point $\psi$ belongs to $P S H(X \backslash E, \omega)$, satisfies

$$
(\omega+\sqrt{-1} \partial \bar{\partial} \psi)^{n}=\alpha^{n} \Omega
$$

on $X \backslash E$, and is bounded near $E$. It follows that $\psi$ extends to a bounded function in $\operatorname{PSH}(X, \omega)$ and the above Monge-Ampère equation holds on $X$ because the Borel measure $(\omega+\sqrt{-1} \partial \bar{\partial} \psi)^{n}$ does not charge the analytic set $E$. Then by the uniqueness part of Theorem 2.1 of [EGZ], we must have $\psi=\varphi$. This implies that $\varphi_{t} \rightarrow \varphi$ in $C^{\infty}$ on compact subsets of $X \backslash E$, and that $\varphi$ is smooth there.

\section{Examples}

In this section we will give some examples where Theorem 1.1 applies. The constructions are well-known and come from algebraic geometry.

Let us look at the case $n=2$ first, the case $n=1$ being trivial. The only projective Calabi-Yau surfaces are tori, bi-elliptic, Enriques and $K 3$ surfaces (recall that the Calabi Conjecture has been successfully applied to the study of $K 3$ surfaces by Todorov [To] and Siu [Si1]). If $X$ is a torus and $L$ is a nef and big line bundle on $X$, then $L$ is ample, and so Theorem 1.1 is vacuous in this case. Similarly if $X$ is bi-elliptic, then $X$ is a finite unramified quotient of a torus, so a nef and big line bundle on $X$ pulls back to a nef and 
big line bundle on a torus. But this must be ample, and so the original line bundle is ample too (Corollary 1.2.28 in $[\mathrm{L}]$ ) and Theorem 1.1 is again empty. If $X$ is an Enriques surface, then $X$ is an unramified $2: 1$ quotient of a $K 3$ surface, so the study of Ricci-flat metrics on $X$ is reduced to the case of a $K 3$ surface. Finally, let us see that there exist projective $K 3$ s that admit a nef and big line bundle that is not ample, to which Theorem 1.1 applies. For example, let $Y$ be the quotient surface $T / i$ where $T$ is the standard torus $\mathbb{C}^{2} / \mathbb{Z}^{4}$ and $i$ is induced by the involution $i(z, w)=(-z,-w)$ of $\mathbb{C}^{2}$. The surface $Y$ has 16 singular points, which are rational double points, and is a Calabi-Yau model. Blowing up these 16 points gives a smooth projective $K 3$ surface $X$ (called a Kummer surface), and we can take $L$ to be the pullback of any ample divisor on $Y$. The set $E$, being equal to the null locus of $L$, is readily seen to be the union of the 16 exceptional divisors, which are $(-2)$ curves. Then Theorem 1.1 applies, and the limit of smooth Ricci-flat metrics on $X$ with classes approaching $c_{1}(L)$ is the pullback of the unique Ricci-flat (actually flat) orbifold Kähler metric on $Y$ in the given class. This originally appeared as Theorem 8 in [KT].

Now we show that conversely all examples of Theorem 1.1 on $K 3$ surfaces with $\alpha=c_{1}(L)$ are of the form $f: X \rightarrow Y$ where $Y$ is an orbifold $K 3$ surface, $k L=f^{*} A$, for some $k \geq 1$ and some ample divisor $A$ on $Y$. Let $X$ be a projective $K 3$ surface and $L$ a nef and big line bundle on $X$. By Theorem 2.1 we know that some power $k L$ is globally generated, and we might as well assume that $k=1$. Then the contraction map $f$ of $L$ contracts an irreducible curve $C$ to a point if and only if $C \cdot L=0$. But since $L \cdot L>0$, the Hodge index theorem implies that $C \cdot C<0$. The long exact sequence in cohomology associated to the sequence

$$
0 \rightarrow \mathcal{O}_{X}(-C) \rightarrow \mathcal{O}_{X} \rightarrow \mathcal{O}_{C} \rightarrow 0
$$

implies that $H^{1}(X, \mathcal{O}(-C))=0$. Serre duality on the other hand gives $H^{2}(X, \mathcal{O}(C))=$ $H^{0}(X, \mathcal{O}(-C))=0$, and $H^{1}(X, \mathcal{O}(C))=H^{1}(X, \mathcal{O}(-C))=0$. Riemann-Roch then yields

$$
\operatorname{dim} H^{0}(X, \mathcal{O}(C))=2+\frac{1}{2} C \cdot C,
$$

which implies that $C \cdot C$ must be even. But since $\pi(C)=(C \cdot C) / 2+1$, the virtual genus of $C$, is nonnegative, we see that $C \cdot C=-2$. This implies that $\pi(C)=0$ and so $C$ is a smooth rational curve with self-intersection -2 . Then the point $f(C)$ is a rational double point, and so $Y=f(X)$ is an orbifold $K 3$ surface. Notice that Ricci-flat orbifold metrics on $Y$ exist by [Y'], [Kob].

Now we turn to examples in dimension 3. The first one is known as conifold in the physics literature [GMS], and is described in detail in Section 1.2 of [Ro], for example. Roughly speaking, it is a 3-dimensional Calabi-Yau model $Y$ that sits in $\mathbb{P}^{4}$ as a nodal quintic. It has 16 singular points, which are nodes and not of orbifold type. Moreover, there exists a small resolution $f: X \rightarrow Y$, which is a birational morphism with $X$ a smooth Calabi-Yau threefold, that is an isomorphism outside the preimages of the nodes, which are 16 rational curves. If $L$ is the pullback of any ample divisor on $Y$, then $L$ is nef and big on $X$, and the limit of smooth Ricci-flat metrics on $X$ with classes approaching $c_{1}(L)$ is the pullback of the unique singular Ricci-flat metric on $Y$, which exists by [EGZ]. 
The convergence is smooth on compact sets outside the union of the 16 exceptional curves (which is clearly equal to the null locus of $L$ ). There are also other 3-dimensional examples where the singularities of $Y$ are not isolated: one of these is described in Example 4.6 in [W1], and $Y$ has a curve $C$ of singularities. Blowing up $C$ gives a Calabi-Yau threefold $X$; if $L$ is the pullback of any ample divisor on $Y$, then the null locus of $L$ is the exceptional divisor $S$ which is a smooth surface ruled over $C$. Again our Theorem 1.1 applies, and the convergence is smooth off $S$.

\section{Further directions}

First let us mention an interesting question that arises from Theorem 1.1. We know that on $X \backslash E$ the Ricci-flat metrics converge smoothly on compact sets to an incomplete Ricciflat metric $\omega_{1}$. Its metric completion is a metric space $\left(X_{\infty}, d_{\infty}\right)$. Do the original metrics $\left(X, \omega_{t}\right)$ actually converge to $\left(X_{\infty}, d_{\infty}\right)$ in the Gromov-Hausdorff topology? We can prove this in the case when $X$ is a $K 3$ surface and $\alpha=c_{1}(L)$. In fact, from Section 5 we know that $E$ is a union of (-2)-curves and the contraction map $f: X \rightarrow Y$ maps them to orbifold points. The results of $[\overline{\mathrm{An}}],[\mathrm{BKN}]$, [Ti] imply that a subsequence of $\left(X, \omega_{t}\right)$ converges to $Y$ with its orbifold Ricci-flat metric $d_{\infty}$ in the Gromov-Hausdorff topology. But on the smooth part of $Y$ the metrics $\omega_{1}$ and $d_{\infty}$ coincide, because they are both singular Ricci-flat metrics on the whole of $Y$. Hence the metric completion of $\omega_{1}$ is $d_{\infty}$.

Also, when $X$ admits a birational Calabi-Yau model $Y$, which has a singular Ricci-flat metric by [EGZ], what is the relation between $Y$ and $X_{\infty}$ ?

There are two possible directions where it would be desirable to extend Theorem 1.1 The first case is when we look at the whole Kähler cone, instead of just the ample cone, and possibly drop the projectiveness assumption. Suppose $X$ is a compact Calabi-Yau $n$-fold and fix a Ricci-flat metric $\omega_{0}$ on $X$. The Néron-Severi space $N^{1}(X)_{\mathbb{R}}$ embeds into

$$
H_{\mathbb{R}}^{1,1}(X)=H^{2}(X, \mathbb{R}) \cap H^{1,1}(X),
$$

but in general it is a proper subspace (for example a generic projective $K 3$ has $\rho(X)=$ $\left.1<20=\operatorname{dim} H_{\mathbb{R}}^{1,1}(X)\right)$. Inside $H_{\mathbb{R}}^{1,1}(X)$ we have $\mathcal{K}$, the Kähler cone, and its closure $\overline{\mathcal{K}}$, the nef cone. We have

$$
\mathcal{K}_{N S}=\mathcal{K} \cap N^{1}(X)_{\mathbb{R}}
$$

and similarly for the nef cone. Given a nonzero class $\alpha \in \overline{\mathcal{K}} \backslash \mathcal{K}$, and a smooth path $\alpha_{t}:[0,1] \rightarrow \overline{\mathcal{K}}$ that ends at $\alpha$, Yau's Theorem gives a path $\omega_{t}$ of Ricci-flat metrics in $\alpha_{t}$ and we can analyze their behavior as $t$ approaches 1 . Let us assume that $\alpha$ is big, which again means that $\alpha^{n}>0$. We would like to repeat the construction we did in the algebraic case. There are two main points where we used the assumption that $X$ was projective and that $\alpha$ belonged to the Néron-Severi space: Proposition 4.1 and Kodaira's lemma. We conjecture that the analogue of Proposition 4.1 still holds, namely we propose 
Conjecture 6.1. Let $X$ be a compact Kähler Calabi-Yau manifold, and $\alpha \in H_{\mathbb{R}}^{1,1}(X)$ be a class which is nef and big, but not Kähler. Then $\alpha$ can be represented by a smooth $(1,1)$ form $\omega$ which is pointwise nonnegative and which is Kähler outside a proper analytic subvariety $E \subset X$.

Notice that the proof of this conjecture would have to use the fact the $X$ is Calabi-Yau, since in general a nef and big class cannot be represented by a smooth nonnegative form (see Example 3.15 in [BB] which is based on [DPS2]). If this conjecture were proved, we could then write $\omega$ as the smooth limit of Kähler forms in $\alpha_{t}$, as in Proposition 4.1 The correct substitute for Kodaira's lemma would then be given by the theory of closed positive currents: following [D2], which relies on the fundamental [DP], we know that there would exist a modification $\pi: \tilde{X} \rightarrow X$ such that

$$
\pi^{*} \omega=\tilde{\omega}+[E]-\sqrt{-1} \partial \bar{\partial} \eta
$$

where $\tilde{\omega}$ is a Kähler form on $\tilde{X}, E$ is an effective $\mathbb{Q}$-divisor on $\tilde{X}$ and $\eta$ is quasi-psh, smooth off $E$ and has only $\log$ poles along $E$. Then we could just work on $\tilde{X}$, and get the same estimates as above, outside $E$, thus proving the Kähler analogue of Theorem 1.1

The second direction is to look at the case when the class $\alpha$ is nef but not necessarily big. Notice that Theorem 3.1 gives a uniform diameter bound in this case. A guiding example is the following: let $X$ be an elliptically fibered $K 3$ surface, so $X$ comes equipped with a morphism $f: X \rightarrow \mathbb{P}^{1}$ with fibers elliptic curves. Then the pullback of an ample line bundle on $\mathbb{P}^{1}$ gives a nef line bundle $L$ on $X$ with Iitaka dimension 1 . In the case when all the singular fibers of $f$ are of Kodaira type $I_{1}$, Gross-Wilson have shown in [GW] that sequences of Ricci-flat metrics on $X$ whose class approaches $c_{1}(L)$ converge in $C^{\infty}$ on compact sets of the complement of the singular fibers to the pullback of a Kähler metric on $\mathbb{P}^{1}$. This metric on $\mathbb{P}^{1}$ was first studied by McLean [McL]. In a recent paper, Song-Tian [ST] gave a more direct proof of the result of Gross-Wilson. Moreover, they noticed that McLean's metric satisfies an elliptic equation outside the images of the singular fibers, namely its Ricci curvature equals the pullback of the Weil-Petersson metric from the moduli space of elliptic curves, which comes from the variation of the complex structure of the fibers of $f$.

We believe that in higher dimensions a similar picture should be true, when $\alpha=$ $c_{1}(L)$. In this case Conjecture 2.1 would imply the existence of a morphism $f: X \rightarrow Y$ with connected fibers, where $\operatorname{dim} Y=\kappa(X, L)<n$. Then we expect that outside a proper subvariety $E \subset X$, a sequence of Ricci-flat metrics with class approaching $\alpha$ should converge in $C^{\infty}$ on compact sets of $X \backslash E$ to the pullback of a metric on $Y$. It is readily verified that, up to a subsequence, the Ricci-flat metrics converge weakly as currents to the pullback of a metric on $Y$. The fibers of $f$ are again Calabi-Yau's, and a computation as in [ST] shows that the limit metric on $Y$ will satisfy the same equation as McLean's metric (in this case the potentials of the Ricci-flat metrics have a uniform $C^{0}$ bound [ST]). It might be possible to construct higher-dimensional examples of this behavior using the results of Section 8 in [Fi], where the equation of McLean's metric appears in his condition (C). 
The situation is different when $\alpha$ is not $c_{1}(L)$, and $X$ possibly not projective. Then an example of McMullen [McM] shows that the Ricci-flat metrics can converge smoothly to zero on an open set of $X$. Also easy examples on tori show that the fibration structure as above cannot be expected when the limiting class $\alpha$ is not rational. Instead we still expect the Ricci-flat metrics to converge smoothly on compact sets outside a subvariety $E$ to a limit nonnegative form $\omega$, whose determinant vanishes identically. The kernel of $\omega$ would then define a complex foliation with singularities on $X \backslash E$, whose leaves might be dense in $X$. The leaves of the foliation are always complex submanifolds, but they might not vary holomorphically and the rank of the foliation might change on different open sets (as in McMullen's example). Notice that if the curvature is uniformly bounded, then Ruan's result $[\mathrm{Ru}]$ implies that this picture is basically true and moreover that the foliation is holomorphic, so that its rank is constant on a Zariski open set. In McMullen's example the curvature blows up, and the resulting foliation is not holomorphic, thus showing that Ruan's result does not hold if the curvature is unbounded.

Let us mention that the results of $[\overline{\mathrm{BKN}}],[\mathrm{Ba}]$ also give a description of the behavior of the Ricci-flat metrics near the singularities, where some bubbling occurs. Unfortunately our methods do not seem to give results of this kind and it would be very interesting to study this in higher dimensions when the limit Calabi-Yau model does not have orbifold singularities.

Finally, let us notice that some of the results here generalize to the following setting: $X$ is a compact Kähler manifold, and we fix a smooth volume form $\Omega$. If $\alpha_{t}$ is a path of Kähler classes as at the beginning of this section, then for each $t<1$ Yau's theorem [Y2] gives a unique Kähler form $\omega_{t}$ in $\alpha_{t}$ such that

$$
\omega_{t}^{n}=\frac{\alpha_{t}^{n}}{\int_{X} \Omega} \Omega .
$$

We can then study the behavior of the metrics $\omega_{t}$ as $t$ approaches 1 . If the image of $\alpha_{t}$ lies in $N^{1}(X)_{\mathbb{R}}$ and the limit class $\alpha$ is nef, big and semiample, then the argument of Theorem 1.1 goes through, and we get smooth convergence on compact sets outside a subvariety. Again if $\alpha=c_{1}(L)$ then this subvariety is the null locus of $L$.

Acknowledgments. I would like to thank my advisor Prof. Shing-Tung Yau for suggesting this problem and for constant support. I also thank Prof. Curt McMullen and Prof. M. S. Narasimhan for inspiring conversations, Chen-Yu Chi, Jian Song, Gábor Székelyhidi and Ben Weinkove for useful comments, and the referee for some interesting suggestions.

Part of this work was carried out while the author was visiting the Morningside Center of Mathematics in Beijing; the author is supported in part by a Harvard Merit Fellowship.

\section{References}

[An] Anderson, M. T.: Ricci curvature bounds and Einstein metrics on compact manifolds. J. Amer. Math. Soc. 2, 455-490 (1989) Zbl 0694.53045 MR 0999661

[Au] Aubin, T.: Équations du type Monge-Ampère sur les variétés kähleriennes compactes. C. R. Acad. Sci. Paris Sér. A-B 283, Aiii, A119-A121 (1976) Zbl 0333.53040 MR 0433520 
[Ba] Bando, S.: Bubbling out of Einstein manifolds. Tohoku Math. J. 42, 205-216 (1990); Erratum, Tohoku Math. J. 42, 587-588 (1990) Zbl 0719.53025 MR 1076177

[BKN] Bando, S., Kasue, A., Nakajima, H.: On a construction of coordinates at infinity on manifolds with fast curvature decay and maximal volume growth. Invent. Math. 97, 313-349 (1989) Zbl 0682.53045 MR 1001844

[BHPV] Barth, W. P., Hulek, K., Peters, C. A. M., Van de Ven, A.: Compact Complex Surfaces. Springer, Berlin (2004) Zbl 1036.14016 MR 2030225

[Be] Beauville, A.: Variétés Kähleriennes dont la première classe de Chern est nulle. J. Differential Geom. 18, 755-782 (1983) Zbl 0537.53056 MR 0730926

[BT] Bedford, E., Taylor, B. A.: The Dirichlet problem for a complex Monge-Ampère equation. Invent. Math. 37, 1-44 (1976) Zbl 0315.31007 MR 0445006

[BB] Berman, R., Boucksom, S.: Capacities and weighted volumes of line bundles. Preprint, arXiv:0803.1950v1

[CL] Cascini, P., La Nave, G.: Kähler-Ricci flow and the Minimal Model Program for projective varieties. Preprint, arXiv:math/0603064

[CCT] Cheeger, J., Colding, T. H., Tian, G.: On the singularities of spaces with bounded Ricci curvature. Geom. Funct. Anal. 12, 873-914 (2002) Zbl 1030.53046 MR 1937830

[Cr] Croke, C. Some isoperimetric inequalities and eigenvalue estimates. Ann. Sci. École Norm. Sup. 13, 419-435 (1980) Zbl 0465.53032 MR 0608287

[D] Demailly, J.-P.: Monge-Ampère operators, Lelong numbers and intersection theory. In: Complex Analysis and Geometry, Plenum, New York, 115-193 (1993) Zbl 0792.32006 MR 1211880

[DP] Demailly, J.-P., Păun, M.: Numerical characterization of the Kähler cone of a compact Kähler manifold. Ann. of Math. 159, 1247-1274 (2004) Zbl 1064.32019 MR 2113021

[DPS1] Demailly, J.-P., Peternell, T., Schneider, M.: Kähler manifolds with numerically effective Ricci class. Compos. Math. 89, 217-240 (1993) Zbl 1008.32008 MR 1255695

[DPS2] Demailly, J.-P., Peternell, T., Schneider, M.: Compact complex manifolds with numerically effective tangent bundles. J. Algebraic Geom. 3, 295-345 (1994) Zbl 0827.14027 MR 1257325

[DZ] Dinew, S., Zhang, Z.: Stability of bounded solutions for degenerate complex MongeAmpère equations. Preprint, arXiv:0711.3643v2

[EGZ] Eyssidieux, P., Guedj, V., Zeriahi, A.: Singular Kähler-Einstein metrics. Preprint, arXiv:math/0603431

[Fi] Fine, J.: Constant scalar curvature Kähler metrics on fibred complex surfaces. J. Differential Geom. 68, 397-432 (2004) Zbl 1085.53064 MR 2144537

[FM] Fong L.-Y., McKernan, J.: Log abundance for surfaces. In: Flips and Abundance for Algebraic Threefolds, Astérisque 211, 127-137 (1992) Zbl 0807.14029

[GMS] Greene, B. R., Morrison, D. R., Strominger, A.: Black hole condensation and the unification of string vacua. Nuclear Phys. B 451, 109-120 (1995) Zbl 0908.53041 MR 1352415

[Gr] Gromov, M.: Metric Structures for Riemannian and Non-Riemannian Spaces. Birkhäuser, Boston (1999) Zbl 0953.53002 MR 1699320

[GW] Gross, M., Wilson, P. M. H.: Large complex structure limits of $K 3$ surfaces. J. Differential Geom. 55, 475-546 (2000) Zbl 1027.32021 MR 1863732

[HM] Hacon, C., McKernan, J.: On the existence of flips. Preprint, arXiv:math/0507597

[HW] Headrick, M., Wiseman, T.: Numerical Ricci-flat metrics on K3. Classical Quantum Gravity 22, 4931-4960 (2005) Zbl 1085.53035 MR 2194329 
[Ka1] Kawamata, Y.: Crepant blowing-up of 3-dimensional canonical singularities and its application to degenerations of surfaces. Ann. of Math. 127, 93-163 (1988) Zbl 0651.14005 MR 0924674

[Ka2] Kawamata, Y.: On the cone of divisors of Calabi-Yau fiber spaces. Int. J. Math. 8, 665687 (1997) Zbl 0931.14022 MR 1468356

[KMM] Kawamata, Y., Matsuda, K, Matsuki, K.: Introduction to the minimal model problem. In: Algebraic Geometry (Sendai, 1985), Adv. Stud. Pure Math. 10, North-Holland, Amsterdam, 283-360 (1987) Zbl 0672.14006 MR 0946243

[Kob] Kobayashi, R.: Einstein-Kähler $V$-metrics on open Satake $V$-surfaces with isolated quotient singularities. Math. Ann. 272, 385-398 (1985) Zbl 0556.14019 MR 0799669

[KT] Kobayashi, R., Todorov, A. N.: Polarized period map for generalized $K 3$ surfaces and the moduli of Einstein metrics. Tohoku Math. J. 39, 341-363 (1987) Zbl 0646.14029 MR 0902574

[Koł] Kołodziej, S.: The complex Monge-Ampère equation. Acta Math. 180, 69-117 (1998) Zbl 0913.35043 MR 1618325

[L] Lazarsfeld, R.: Positivity in Algebraic Geometry I \& II. Springer, Berlin (2004) Zbl 1093.14501 1093.14500 MR 2095471 2095472

[Li] Li, P.: On the Sobolev constant and the $p$-spectrum of a compact Riemannian manifold. Ann. Sci. École Norm. Sup. 13, 451-468 (1980) Zbl 0466.53023 MR 0608289

[LY] Li, P., Yau, S.-T.: Estimates of eigenvalues of a compact Riemannian manifold. In: Geometry of the Laplace Operator (Honolulu, Hawaii, 1979), Proc. Sympos. Pure Math. 36, Amer. Math. Soc., Providence, 205-239 (1980) Zbl 0441.58014 MR 0573435

[McL] McLean, R. C.: Deformations of calibrated submanifolds. Comm. Anal. Geom. 6, 705747 (1998) Zbl 0929.53027 MR 1664890

[McM] McMullen, C. T.: Dynamics on $K 3$ surfaces: Salem numbers and Siegel disks. J. Reine Angew. Math. 545, 201-233 (2002) Zbl 1054.37026 MR 1896103

[MP] Miyaoka, Y., Peternell, T.: Geometry of Higher-Dimensional Algebraic Varieties. DMV Seminar 26, Birkhäuser, Basel (1997) Zbl 0865.14018 MR 1468476

[N] Nakamaye, M.: Stable base loci of linear series. Math. Ann. 318, 837-847 (2000) Zbl 1063.14008 MR 1802513

[P1] Păun, M.: On the Albanese map of compact Kähler manifolds with numerically effective Ricci curvature. Comm. Anal. Geom. 9, 35-60 (2001) Zbl 0980.53091 MR 1807951

[P2] Păun, M.: Regularity properties of the degenerate Monge-Ampère equations on compact Kähler manifolds. Preprint, arXiv:math/0609326

[Ro] Rossi, M.: Geometric transitions. J. Geom. Phys. 56, 1940-1983 (2006) Zbl 1106.32019 MR 2240431

[Ru] Ruan, W.-D.: On the convergence and collapsing of Kähler metrics. J. Differential Geom. 52, 1-40 (1999) Zbl 1039.53045 MR 1743466

[SY] Schoen, R., Yau, S.-T. Lectures on Differential Geometry. Int. Press, Cambridge, MA (1994) Zbl 0830.53001 MR 1333601

[Sh] Shokurov, V. V.: 3-fold log models. J. Math. Sci. 81, 2667-2699 (1996) Zbl 0873.14014 MR 1420223

[Si1] Siu, Y.-T.: Every $K 3$ surface is Kähler. Invent. Math. 73, 139-150 (1983) Zbl 0557.32004 MR 0707352

[Si2] Siu, Y.-T.: Lectures on Hermitian-Einstein Metrics for Stable Bundles and KählerEinstein Metrics. DMV Seminar 8, Birkhäuser, Basel (1987) Zbl 0631.53004 MR 0904673

[ST] Song, J., Tian, G.: The Kähler-Ricci flow on surfaces of positive Kodaira dimension. Invent. Math. 170, 609-653 (2007) Zbl 1134.53040 MR 2357504 
[Ti] Tian, G.: On Calabi's conjecture for complex surfaces with positive first Chern class. Invent. Math. 101, 101-172 (1990) Zbl 0716.32019 MR 1055713

[TZ] Tian, G., Zhang, Z.: On the Kähler-Ricci flow on projective manifolds of general type. Chinese Ann. Math. Ser. B 27, 179-192 (2006) Zbl 1102.53047 MR 2243679

[To] Todorov, A. N.: Applications of the Kähler-Einstein-Calabi-Yau metric to moduli of $K 3$ surfaces. Invent. Math. 61, 251-265 (1980) Zbl 0472.14006 MR 0592693

[Ts] Tsuji, H.: Existence and degeneration of Kähler-Einstein metrics on minimal algebraic varieties of general type. Math. Ann. 281, 123-133 (1988) Zbl 0631.53051 MR 0944606

[W1] Wilson, P. M. H.: The Kähler cone on Calabi-Yau threefolds. Invent. Math. 107, 561-583 (1992) Zbl 0766.14035 MR 1150602

[W2] Wilson, P. M. H.: Metric limits of Calabi-Yau manifolds. In: The Fano Conference. Univ. Torino, Turin, 793-804 (2004) Zbl 1061.32019 MR 2112603

[Y1] Yau, S.-T.: Calabi's conjecture and some new results in algebraic geometry. Proc. Nat. Acad. Sci. U.S.A. 74, 1798-1799 (1977) Zbl 0355.32028 MR 0451180

[Y2] Yau, S.-T.: On the Ricci curvature of a compact Kähler manifold and the complex MongeAmpère equation, I. Comm. Pure Appl. Math. 31, 339-411 (1978) Zbl 0369.53059 MR 0480350

[Y3] Yau, S.-T.: Problem section. In: Seminar on Differential Geometry. Ann. of Math. Stud. 102, Princeton Univ. Press, 669-706 (problem 49) (1982) Zbl 0479.53001 MR 0645762

[Y4] Yau, S.-T.: Open problems in geometry. In: Differential Geometry: Partial Differential Equations on Manifolds, Proc. Sympos. Pure Math. 54, Part 1, Amer. Math. Soc., 1-28 (problem 88) (1993) Zbl 0801.53001 MR 1216573

[Z1] Zhang, Z.: On degenerate Monge-Ampère equations over closed Kähler manifolds. Int. Math. Res. Notices 2006, no. 11, art. ID 63640, 18 pp. Zbl 1112.32021 MR 2233716

[Z2] Zhang, Z.: Degenerate Monge-Ampère equations over projective manifolds. Ph.D. thesis, MIT, 2006 